\title{
Simulating chalcogen bonding using molecular mechanics: A pseudoatom approach to model ebselen.
}

\author{
Thomas Fellowes, Jonathan M. White
}

May 21, 2020

\begin{abstract}
The organoselenium compound ebselen has recently been investigated as a treatment for COVID-19, however efforts to model ebselen in silico have been hampered by the lack of a efficient and accurate method to assess its binding to biological macromolecules. We present here a Generalized Amber Force Field modification which incorporates classical parameters for the selenium atom in ebselen, as well as a positively charged pseudoatom to simulate the $\sigma$-hole, a quantum mechanical phenomenon that dominates the chemistry of ebselen. Our approach is justified using an energy decomposition analysis of a number DFT optimised structures, which shows that the $\sigma$-hole interaction is primarily electrostatic in origin. Finally, our model is verified by conducting MD simulations on a number of simple complexes, as well the clinically relevant SOD1, which is known to bind to ebselen.
\end{abstract}

\section{Introduction}

Ebselen (1) is a molecule that has piqued the interest of many medicinal chemists, in no small part due to its decidedly non-druglike appearance. First synthesized in 1924, its unusual properties went more or less uninvestigated for more than 50 years. ${ }^{1}$ Interest in ebselen boomed in the early 1980s, and since then it has been the subject of several studies into its synthesis, biological properties, and metabolism. ${ }^{2-10}$ Its biological activity can be broadly attributed to its ability to neutralise reactive oxygen species, reducing the level of oxidative stress to which cells are subjected. ${ }^{11}$ To this end, ebselen has been investigated for its neuroprotective, mood-stabilising, anti-inflammatory, and anti-cancer properties. ${ }^{12-17}$ Very recently it was identified as a compound of interest for the treatment of COVID-19, showing promising inhibition of the viral $\mathrm{M}^{\text {pro }}$ protease enzyme. ${ }^{18}$

The in vivo antioxidant ability of ebselen is believed to be mediated through a catalytic cycle analogous to that of glutathione peroxidase (a selenoenzyme). ${ }^{19}$ The selenium-containing heterocycle is reductively opened to afford the free selenol 1a, which is the active catalyst. This is rapidly oxidised by ROS to a selenenic acid $\mathbf{2}$, which is then reduced back to the $\mathbf{1 a}$ by glutathione (GSH) via a selenenyl sulfide 3. Its activity against a number of other targets appears to also be mediated through formation of a covalent complex via nucleophilic attack at the selenium. There is also evidence that ebselen interacts with targets non-covalently. ${ }^{18}$ These interactions may include association with aromatic or hydrophobic residues, or H-bonding through the carbonyl. Ebselen can also form non-covalent complexes with Lewis bases through an electrophilic $\sigma$-hole on the selenium atom. ${ }^{20,21}$

Molecular modelling is a vital tool in drug development, allowing for rapid and broad-reaching screening of drug candidates against likely substrates at minimal cost and risk. Ab initio quantum methods (QM) are widely used to model small molecules (generally smaller than a few hundred atoms), where 


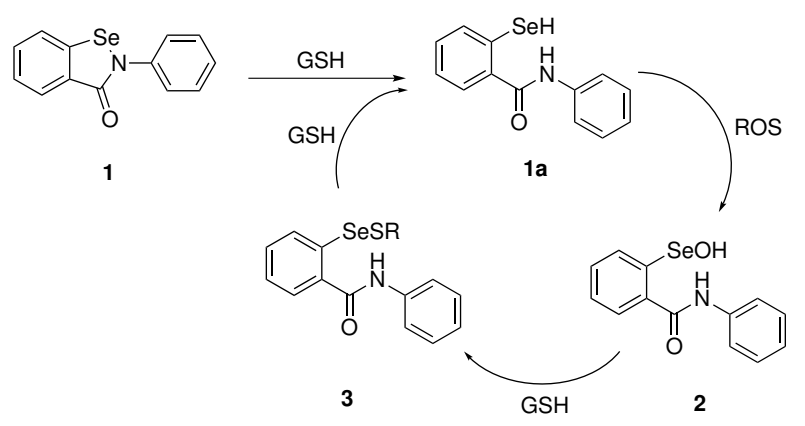

their accuracy and ability to describe quantum effects underlying photochemical properties and bond breaking/formation processes are critical. They are, however, computationally costly. Molecular mechanics (MM), where systems are treated strictly classically, is a viable alternative for large systems. The drawbacks are that MM relies on having an extensive parameter set (a "force field") to describe the system (which is not necessarily available, or applicable to the system at hand), and that descriptions of quantum effects often fail spectacularly.

Both of these issues are encountered when attempting to model ebselen using MM. Firstly, parameters to describe selenium-containing small molecules are simply not available in most popular force fields, including GAFF, Gromos, and CGenFF. This has been substantially addressed in the work of Torsello et al, where extensive parametrization of a series of diaryl diselenides and diaryl ditellurides was performed, and they provided a methodology to extend this to general chalcogen-containing molecules. ${ }^{22}$ They did not, however, address the second issue of quantum effects, which is reasonable given that they do not play a large role in diselenides. The chemistry of ebselen, on the other hand, is dominated by the $\sigma$-hole, which is a quantum effect. ${ }^{20}$ The $\sigma$-hole is a region of positive electrostatic potential situated opposite to the $\mathrm{Se}-\mathrm{N}$ bond, caused by a strongly anisotropic electron distribution around the selenium atom. This causes the selenium to adopt a highly directional electrophilic character, which can lead to the formation of "chalcogen-bonds" with electron pair donors (named by analogy to the ubiquitous hydrogen bond). ${ }^{23}$

The presence of a $\sigma$-hole is not a new problem in MM, nor are they exclusive to chalcogens, as they are also found on the heavier halogens, where they give rise to halogen bonding. ${ }^{24}$ Perhaps due to the higher prevalence of halogens in drug-like molecules, a number of approaches have been proposed to account for $\sigma$-holes in halogenated molecules. The common theme in these methods is the inclusion of a pseudoatom with positive electrostatic potential attached to the halogen atom. This pseudoatom is variously called an extra point (EP), explicit $\sigma$-hole (ESH), or virtual or off-atom centered point, and the approaches differ in the location of the pseudoatom and method used to derive its charge. ${ }^{25-28}$ Lone pairs can also be described using negatively charged pseudoatoms, and this approach has been used for some time. ${ }^{28-30}$

It is worth noting an alternative approach, used by Cozzolino and Vargas-Baca, which treats secondary bonding interactions as true bonds, with an explicitly parametrized potential. ${ }^{31}$ This was used to parametrize the supramolecular synthon 1,2,5-telluradizaole, which is known to self assemble into a range of interesting structures. This approach, however, relies on describing the bond using an anharmonic potential, which is not easily implemented in AMBER.

The inability to model ebselen in biological systems is a major hurdle in understanding the mechanism of its action. In this work we develop a parameter set for the selenium atom in ebselen, including a pseudoatom to simulate the $\sigma$-hole. This work is implemented in AMBER, due to its popularity, speed, and robustness, although there is no reason these parameters could not be extended to other force fields. 


\begin{tabular}{ccc}
\hline Parameter & $x_{0}$ & $k$ \\
\hline $\mathrm{r}(\mathrm{Se}-\mathrm{N})$ & 1.8586 & 434.67 \\
$\mathrm{r}(\mathrm{Se}-\mathrm{C})$ & 1.8829 & 422.33 \\
$\angle(\mathrm{C}-\mathrm{Se}-\mathrm{N})$ & 86.6 & 610.7 \\
$\angle\left(\mathrm{Se}-\mathrm{N}-\mathrm{C}_{\mathrm{ar}}\right)$ & 119.6 & 182.7 \\
$\angle\left(\mathrm{Se}-\mathrm{N}-\mathrm{C}_{\mathrm{CO}}\right)$ & 115.8 & 404.5 \\
$\angle(\mathrm{C}-\mathrm{C}-\mathrm{Se})$ & 119.4 & 329.2 \\
\hline
\end{tabular}

Table 1: Classical parameters for ebselen. Bond lengths are given in $\AA$, and angles in degrees. Force constants are given in $\mathrm{kcal} / \mathrm{mol} \cdot \AA^{2}$ or $\mathrm{kcal} / \mathrm{mol} \cdot \mathrm{radian}^{2}$.

\begin{tabular}{ccccccccc}
\hline Parameter & $\begin{array}{c}V_{\max , 2} \\
\mathrm{kcal} / \mathrm{mol}\end{array}$ & $\begin{array}{c}V_{\max , 4} \\
\mathrm{kcal} / \mathrm{mol}\end{array}$ & $\begin{array}{c}V_{\max , 6} \\
\mathrm{kcal} / \mathrm{mol}\end{array}$ & $\begin{array}{c}V_{\max , 8} \\
\mathrm{kcal} / \mathrm{mol}\end{array}$ & $\begin{array}{c}\gamma_{2} \\
{ }^{\circ}\end{array}$ & $\begin{array}{c}\gamma_{4} \\
{ }^{\circ}\end{array}$ & $\begin{array}{c}\gamma_{6} \\
{ }^{\circ}\end{array}$ & $\begin{array}{c}\gamma_{8} \\
{ }^{\circ}\end{array}$ \\
\hline$\phi\left(\mathrm{C}_{\mathrm{ar}}-\mathrm{C}_{\mathrm{ar}}-\mathrm{N}-\mathrm{Se}\right)$ & -0.0862 & 0.7841 & 0.0363 & 0.0424 & 180 & 0 & 0 & 0 \\
\hline
\end{tabular}

Table 2: Dihedral parameters for ebselen.

We also restrict ourselves to the "vanilla" feature set of AMBER, given that advanced features such as polarizable force fields are not widely used in the analysis of ligand-receptor systems. We then show that this model accurately reproduces experimental geometries and energies, and compares favourably to ab initio calculations. This force field will prove useful in understanding the interactions between ebselen and current targets, and possibly lead to the discovery of new targets.

\section{Results and Discussion}

We begin by deriving the classical bonding parameters involving selenium in ebselen, using the procedure of Torsello. ${ }^{22}$ All quantum calculations were performed using Gaussian09, unless otherwise specified. ${ }^{32}$ The ground state geometry of ebselen was optimised at the $\omega$ B97X-D/def2TZVP level, followed by vibrational analysis to confirm the structure was minimised. ${ }^{33-35}$ Partial charges were assigned to the atoms using the RESP scheme, at the HF $/ 6-31 \mathrm{G}^{*}$ level. ${ }^{36}$ This was chosen for consistency with existing AMBER force fields.

Bond and angle force constants were derived by conducting a relaxed potential energy surface scan over a range of $\pm 0.3 \AA$ for bonds and $\pm 10^{\circ}$ for angles. The resulting data was truncated to within $5 \mathrm{kcal} / \mathrm{mol}$ of the equilibrium energy (at larger distances the surfaces were appreciably anharmonic), and this surface was fitted with a classical harmonic oscillator model (equation 1) using the nls function in the $\mathrm{R}$ software package. ${ }^{37}$ The equilibrium distance/angle $x_{0}$ was fixed to the value from the optimised geometry. Torsion angles were similarly scanned, except the resulting surfaces were fit using a periodic series truncated to the fourth order (equation 2). The resulting parameters are presented in tables 1 and 2 .

$$
\begin{gathered}
V(x)=\frac{1}{2} k\left(x-x_{0}\right)^{2} \\
V(\phi)=\sum_{n=1}^{4}\left(\frac{V_{\max , n}}{2} \times\left(1+\cos \left(n \phi+\gamma_{n}\right)\right)\right)
\end{gathered}
$$

Electrostatic potentials were calculated using the cubegen program in the Gaussian suite, or mol2cub (https://github.com/tjfellowes/mol2cub).

Values of 2.12 and 0.2910 for the Lennard-Jones parameters $\sigma$ and $\varepsilon$ were used for selenium. Default 


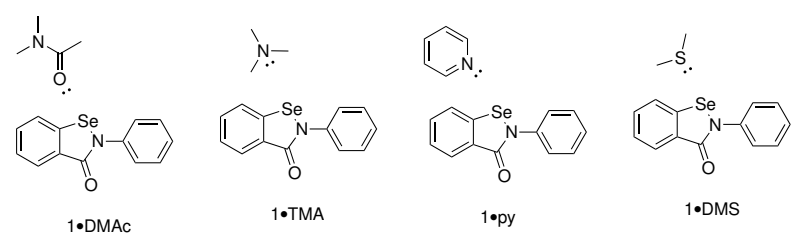

Figure 1: Structures of complexes used for SAPT(DFT) analysis.

GAFF values were used for all other atoms, and Lorentz/Berthelot mixing rules were used to derive cross-terms.

While attempting to model the $\sigma$-hole using molecular mechanics, we must remember that we are forcing a classical treatment onto an inherently quantum phenomenon. That said, some parts of the quantum phenomenon are easier than others to treat classically. There are thought to be three attractive energetic components which contribute to a $\sigma$-hole interaction. Namely, electrostatics, induction, and dispersion. ${ }^{38-40}$ The magnitudes of each component of $\sigma$-hole interactions has been the subject of vigorous debate in recent years. For many applications, these disagreements are fairly philosophical and of little consequence, however this is not the case when attempting to model $\sigma$-hole interactions using MM.

The electrostatic component generally refers to the interaction between two static (not distorted by each other) electric fields, which can be graphically represented by visualising the electrostatic potential surfaces of the donor and acceptor moieties (figure 3). This is already treated in MM (for the case of atom centered charges) as a sum of pairwise interactions. The accuracy of this component is only limited by the resolution of the electrostatic potential; it would appear that a pseudoatom approach could thus adequately describe the $\sigma$-hole. Dispersion is accounted for empirically within the $r^{-6}$ term of the Lennard-Jones potential.

Issues arise when attempting to model the induction component of the $\sigma$-hole $E_{\text {ind }}$. This component refers to the redistribution of charge within (polarization) or between (charge-transfer) the donor and acceptor as they approach each other. Movement of charge is simply not accounted for within the most common AMBER force fields (though there has been some progress towards polarizable force fields). This presents a large problem, as charge-transfer drives the strong directionality of $\sigma$-hole interactions, and may account for a significant proportion of their strength.

To ensure that this will not be an insurmountable problem for this parametrization, we conducted energy decomposition analyses (EDA) on a variety of complexes containing ebselen. There are numerous EDA schemes available such as KM-EDA, NEDA, and ALMO, however we chose to use symmetryadapted perturbation theory (SAPT) ${ }^{41,42}$ In contrast to several other schemes, SAPT explicitly includes dispersion (as opposed to adding it as an empirical correction), and contains no physically meaningless "catch-all" energy term. The total interaction energy $E_{\text {tot }}$ is decomposed into an electrostatic component $E_{\text {elst }}$, an inductive component $E_{\text {ind }}$ (this incorporates polarization and charge transfer, as they are not distinct phenomena within the SAPT framework), and a dispersive component $E_{\text {dis }}$. These attractive forces are balanced by a repulsive exchange component $E_{\text {exch }}$.

Four Lewis bases were chosen which are representative of those likely to be encountered in biological systems, and which span a wide range of basicity. Their structures are given in figure 1 . SAPT(DFT) analyses were conducted using the Psi4 software package on geometries optimised at the $\omega$ B97X-D/def2TZVP level, and the results are shown in figure $2 .{ }^{43}$

The SAPT results indicate that the majority (around $80 \%$ ) of the interaction can be described by electrostatics and dispersion. This suggests that the explicit $\sigma$-hole parametrization will be reliable, as 


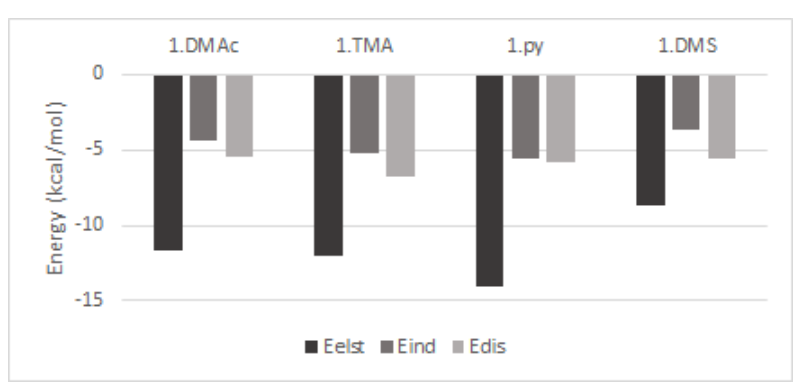

Figure 2: SAPT(DFT) analysis of complexes with four Lewis bases. All energies are given in kcal/mol.

electrostatics and dispersion are relatively well described by MM.

With classical parameters for ebselen in hand, as well as theoretical assurance that the system can be adequately described using electrostatics, we began to optimise parameters for the pseudoatom representing the $\sigma$-hole. While AMBER supports massless extra points (which would be ideal to model the $\sigma$-hole), these are necessarily hardcoded into the program at this stage, and we decided that modification of core routines and recompiling the code was beyond the scope of this work, and represented a significant hurdle to other groups wishing to adopt our model. The mass was therefore set to 3.0 a.m.u. which is not strictly physical, but is necessary to prevent large vibrational excursions. The pseudoatom was intuitively placed on the selenium atom $180^{\circ}$ from the nitrogen, at a distance of $1.32 \AA$. Force constants of $300.0 \mathrm{kcal} / \mathrm{mol} \cdot \AA^{2}$ and $50.0 \mathrm{kcal} / \mathrm{mol} \cdot \mathrm{radian}^{2}$ were applied. The charge was set to $0.21 \mathrm{a} . \mathrm{u}$. (calculated from ESP maps at the $\omega$ B97X-D/def2TZVP level) and RESP was used to construct the remainder of the atomic charges.

The magnitude of the $\sigma$-hole is most sensitive to the distance and charge of the pseudoatom, so these were optimised to reproduce

1. the SAPT(DFT) intermolecular energy, and

2. DFT geometries.

Longer Se-pseudoatom distances were found to give superior results by the first criterion, but caused unstable geometries, with the Lewis base oscillating between extreme angles. This is presumably due to the short range Lennard-Jones repulsion rapidly overwhelming the electrostatic attraction. $1.32 \AA$ was the greatest distance at which this instability was no longer observed at $300 \mathrm{~K}$.

The charge of the pseudoatom was similarly optimised. The value chosen was a compromise between geometry and the electrostatic criteria. 0.21 a.u. gave a Ch-bond distance that was slightly elongated, while the electrostatic potential and energy were noticeably overstated. We attribute this to the small but non-negligible induction/polarization component of the interaction, necessitating an artificial inflation of the electrostatic term to compensate for terms which are not modelled. This is quite evident in the electrostatic potential map (figure 3), however the geometry is still well described.

The Lewis bases chosen for the SAPT(DFT) analysis were constructed in AMBER. GAFF was used for all atoms, and an extra point was added to simulate the lone pair per the method of Dixon and Kollman. ${ }^{29}$ Force constants were reduced as necessary to eliminate unphysical high amplitude vibrations. Geometries were assessed by minimizing the ebselen-Lewis base structure over 1000 cycles, then conducting a $2 \mathrm{~ns} \mathrm{MD}$ trajectory in a vacuum. Trajectories were performed at $300 \mathrm{~K}$ for the strongly bonded systems (1.py and 1.DMAc), however the weaker complexes (1.TMA and 1.DMS) tended to dissociate under these conditions. Their trajectories were therefore conducted at $200 \mathrm{~K}$ and $100 \mathrm{~K}$ respectively. Binding energies were calculated by slowly cooling the system to $0 \mathrm{~K}$, then conducting a short 


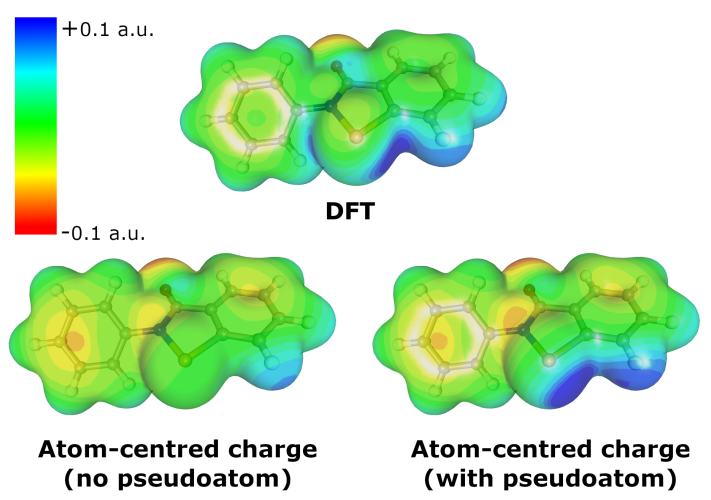

Figure 3: ESP mapped on the 0.005 a.u. electron density isosurface. The $\sigma$-hole is visible as the dark blue region on the DFT and atom-centered charge (with pseudoatom) surfaces.

\begin{tabular}{ccccc}
\hline Complex & $\mathrm{r}(\mathrm{Se} \cdots \mathrm{B})$ & $\angle(\mathrm{N}-\mathrm{Se} \cdots \mathrm{B})$ & $\angle$ lone pair $)$ & $\begin{array}{c}\text { Energy } \\
(\mathrm{kcal} / \mathrm{mol})\end{array}$ \\
\hline $\mathbf{1} \cdot \mathrm{py}$ & $2.98 \AA(2.775 \AA)$ & $169.8^{\circ}\left(176.2^{\circ}\right)$ & $158.2^{\circ}\left(165.7^{\circ}\right)$ & $-8.376(-7.093)$ \\
$\mathbf{1} \cdot \mathrm{DMAc}$ & $2.964 \AA(2.786 \AA)$ & $166.3^{\circ}\left(172.4^{\circ}\right)$ & $129.7^{\circ}\left(117.9^{\circ}\right)$ & $-10.351(-7.551)$ \\
$\mathbf{1} \cdot \mathrm{TMA}$ & $3.091 \AA(2.857 \AA)$ & $167.6^{\circ}\left(177.4^{\circ}\right)$ & $100.71^{\circ}\left(113.6^{\circ}\right)$ & $-6.666(-6.627)$ \\
$\mathbf{1} \cdot \mathrm{DMS}$ & $3.322 \AA(3.265 \AA)$ & $165.0^{\circ}\left(177.4^{\circ}\right)$ & $89.1^{\circ}\left(89.8^{\circ}\right)$ & $-4.541(-5.646)$ \\
\hline
\end{tabular}

Table 3: Median geometric parameters for complexes with 1. DFT equilibrium values are given in brackets for comparison. DFT energies are derived from SAPT(DFT) (table S5).

simulation to determine the potential energy of the system. Relevant parameters are given in figure 4 and table 3, alongside DFT values for comparison.

These results show that Ch-bonds can be adequately described by the inclusion of a positively charged pseudoatom. Interestingly, the weaker complexes (1·TMA and 1·DMS) are better described both in terms of geometry and energy. This may be due to the somewhat decreased charge-transfer component of these interactions, which is poorly described. For the stronger complexes, both the interaction energy and distance are overestimated, representing a compromise between the two criteria.

In order to validate the utility of our model, we conducted a docking experiment with a known ebselen target. Superoxide dismutase-1 (SOD1) forms a covalent complex with ebselen through the Cys111 residue, which appears to support correct folding of the protein, inhibiting aggregation and associated toxicity. ${ }^{44}$ Although formation of the covalent complex cannot be simulated using our model (as this is a bond-forming process), we will be able to visualise the stabilised encounter complex which undergoes ring opening to form the final adduct. Indeed, the Ch-bond formed through the $\sigma$-hole can be thought of as the early stages of a nucleophilic attack at the selenium. ${ }^{20}$ SOD1 (PDB 2C9V) was chosen because of the availability of an atomic resolution structure, demonstrated evidence of ebselen binding, and it's relatively small size. ${ }^{44,45}$ The structure was prepared for AMBER by removing disorder, then removing water and ions (the $\mathrm{Cu}$ and $\mathrm{Zn}$ ions were retained). The ebselen residue was introduced within the binding groove approximately halfway between the two units. The complex was then neutralised by addition of four $\mathrm{Na}^{+}$ions at the sites of most negative electrostatic potential, and solvated with a TIP3P explicit water model to give a final box size of $77.095 \times 96.253 \times 78.411 \AA$. The structure was minimised over 1000 cycles to remove bad contacts, then heated to $300 \mathrm{~K}$ over $200 \mathrm{ps}$. A simulation of 2 ns at $300 \mathrm{~K}$ was then performed to assess the average binding geometry, which was found to exhibit a bifurcated Ch-bond between the expected Cys111 sulfur and the adjacent Ile113 backbone carbonyl (figure 5). A 

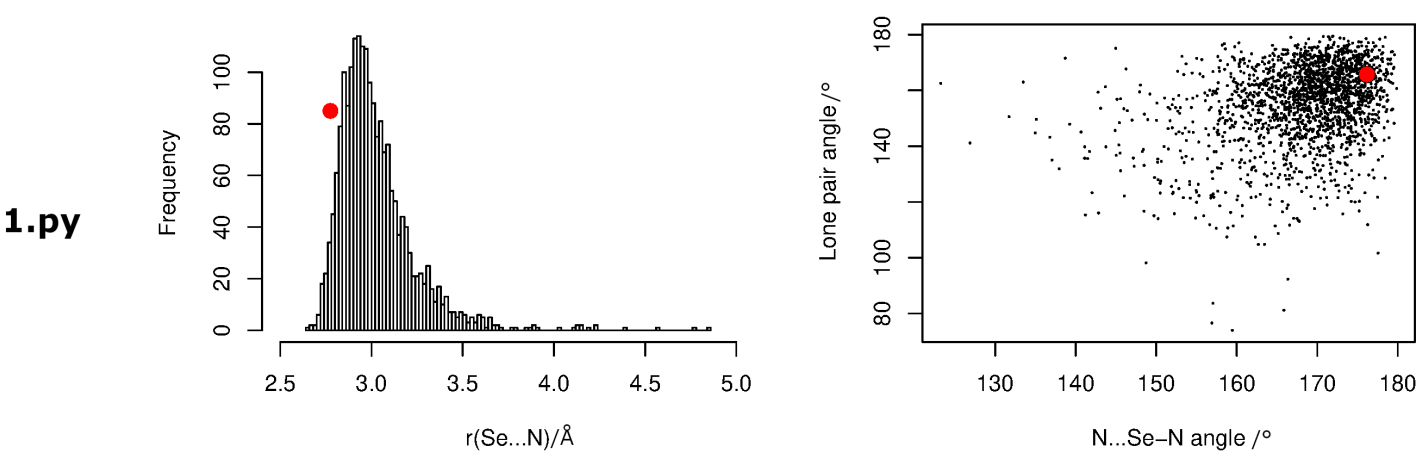

1.DMAc
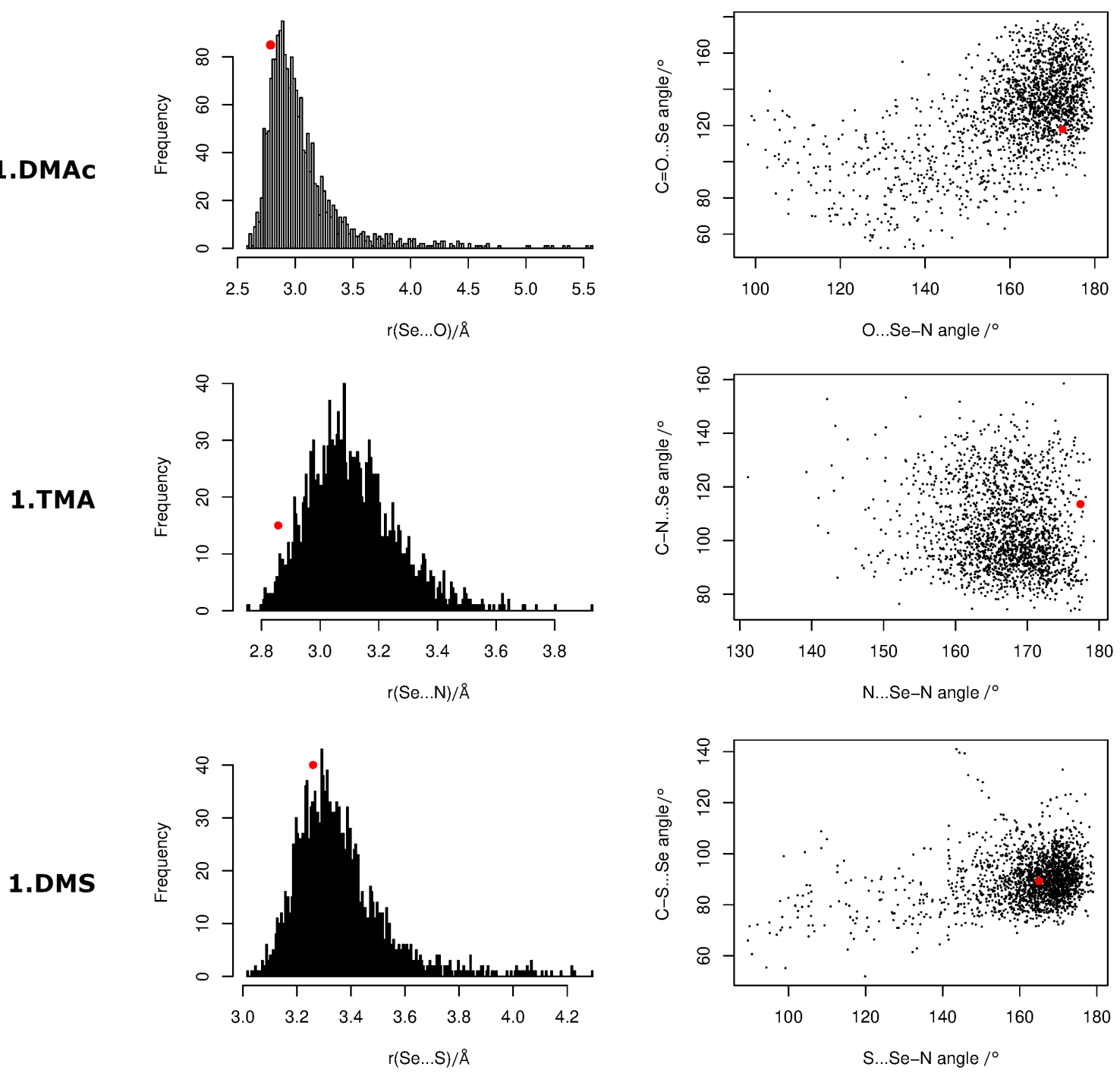

Figure 4: Distribution of geometric parameters for complexes over a 2 ns trajectory. DFT equilibrium values are shown as red circles. 


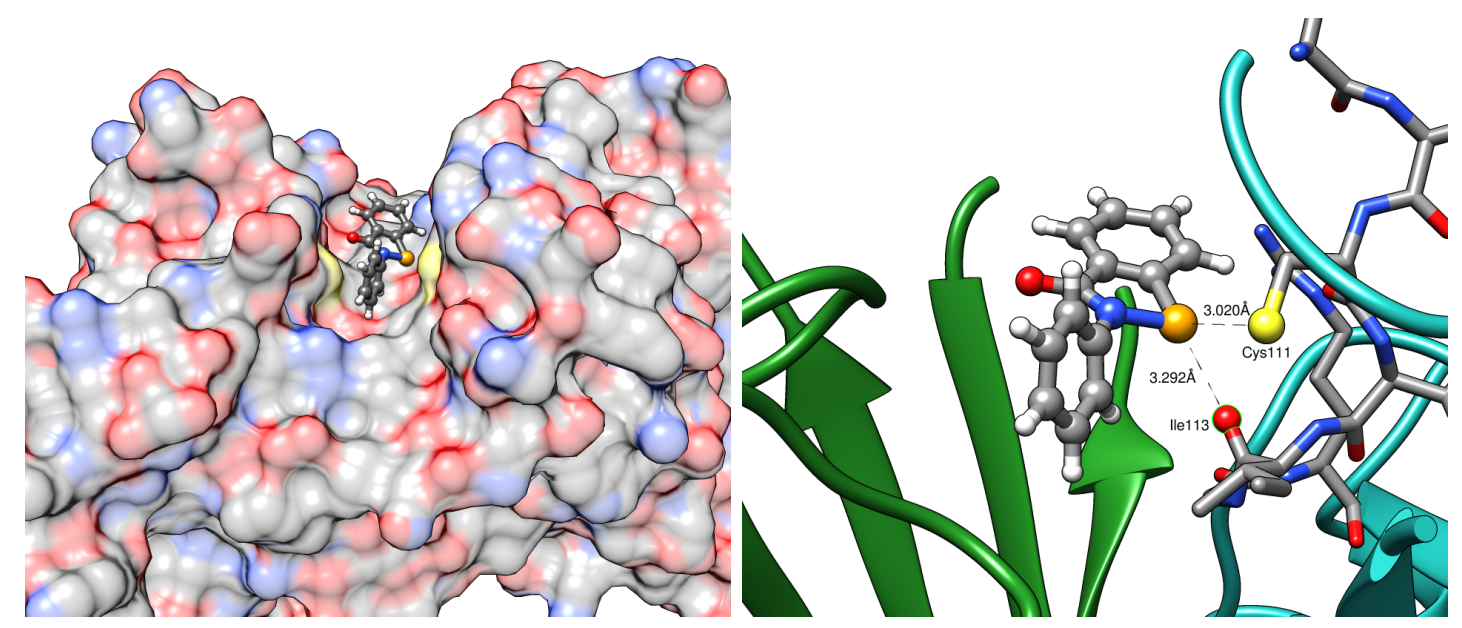

Figure 5: Average binding geometry of ebselen in the SOD1 groove.

similar experiment was performed without the $\sigma$-hole, which failed to bind in a reproducible geometry, with the ebselen molecule wandering through the groove. This is presumably driven by hydrophobic interactions, and the entropic cost of desolvation.

In conclusion, we have developed a set of parameters which can greatly improve modelling of ebselen and its derivatives. Our model gives realistic geometries and energies of gas phase complexes, and reproduces the interaction between ebselen and a protein. Although this work is restricted to ebselen itself, the parameters will be generally applicable to derivatives of ebselen (with appropriate charge fitting), including the selenenic acid $\mathbf{2}$ and selenenyl sulfide $\mathbf{3}$. We hope that these results will be useful for the discovery of new targets.

\section{References}

(1) Lesser, R.; Weiß, R. Berichte der deutschen chemischen Gesellschaft (A and B Series) 1924, 5\%, 1077-1082.

(2) Weber, R.; Renson, M. Bulletin de la Societe Chimique de France 1976, 1124-6.

(3) Renson, M.; Etschenberg, E.; Winkelmann, J. 2-Phenyl-1,2-benzisoselenazol-3(2H)-one containing pharmaceutical preparations and process for the treatment of rheumatic diseases, 1981.

(4) Müller, A.; Cadenas, E.; Graf, P.; Sies, H. Biochemical Pharmacology 1984, 33, 3235-3239.

(5) Wendel, A.; Fausel, M.; Safayhi, H.; Tiegs, G.; Otter, R. Biochemical Pharmacology 1984, 33, $3241-3245$.

(6) Parnham, M. J.; Kindt, S. Biochemical Pharmacology 1984, 33, 3247-3250.

(7) Engman, L.; Hallberg, A. Journal of Organic Chemistry 1989, 54, 2966-2968.

(8) Schewe, T. General Pharmacology 1995, 26, 1153-1169.

(9) Balkrishna, S. J.; Bhakuni, B. S.; Chopra, D.; Kumar, S. Organic Letters 2010, 12, 5394-5397.

(10) Iwasaki, M.; Miki, N.; Tsuchiya, Y.; Nakajima, K.; Nishihara, Y. Organic Letters 2017, 19, 10921095.

(11) Mugesh, G.; Singh, H. B. Chemical Society Reviews 2000, 29, 347-357. 
(12) Parnham, M. J.; Graf, E. Biochemical Pharmacology 1987, 36, 3095-3102.

(13) Kil, J.; Pierce, C.; Tran, H.; Gu, R.; Lynch, E. D. Hearing Research 2007, 226, 44-51.

(14) Singh, N.; Halliday, A. C.; Thomas, J. M.; Kuznetsova, O.; Baldwin, R.; Woon, E. C.; Aley, P. K.; Antoniadou, I.; Sharp, T.; Vasudevan, S. R.; Churchill, G. C. Nature Communications 2013, 4 , 1332-1337.

(15) Azad, G. K.; Tomar, R. S. Molecular Biology Reports 2014, 41, 4865-4879.

(16) Parnham, M.; Sies, H. Expert Opinion on Investigational Drugs 2000, 9, 607-619.

(17) Chantadul, V.; Wright, G. S. A.; Amporndanai, K.; Shahid, M.; Antonyuk, S. V.; Washbourn, G.; Rogers, M.; Roberts, N.; Pye, M.; O’Neill, P. M.; Hasnain, S. S. Communications Biology 2020, 3, 97.

(18) Jin, Z. et al. Nature 2020, DOI: 10.1038/s41586-020-2223-y.

(19) Antony, S.; Bayse, C. A. Inorganic Chemistry 2011, 50, 12075-12084.

(20) Thomas, S. P.; Satheeshkumar, K.; Mugesh, G.; Gururow, T. N. Chemistry - A European Journal 2015, 21, 6793-6800.

(21) Fellowes, T.; White, J. M. CrystEngComm 2019, 21, 1539-1542.

(22) Torsello, M.; Pimenta, A. C.; Wolters, L. P.; Moreira, I. S.; Orian, L.; Polimeno, A. Journal of Physical Chemistry A 2016, 120, 4389-4400.

(23) Murray, J. S.; Lane, P.; Politzer, P. Journal of Molecular Modeling 2009, 15, 723-729.

(24) Clark, T.; Hennemann, M.; Murray, J. S.; Politzer, P. Journal of Molecular Modeling 2007, 13, 291-296.

(25) Rendine, S.; Pieraccini, S.; Forni, A.; Sironi, M. Physical Chemistry Chemical Physics 2011, 13, 19508-19516.

(26) Ibrahim, M. A. A. Journal of Computational Chemistry 2011, 32, 2564-2574.

(27) Kolář, M.; Hobza, P. Journal of Chemical Theory and Computation 2012, 8, 1325-1333.

(28) Harder, E. et al. Journal of Chemical Theory and Computation 2016, 12, 281-296.

(29) Dixon, R. W.; Kollman, P. A. Journal of Computational Chemistry 1997, 18, 1632-1646.

(30) Cieplak, P.; Caldwell, J.; Kollman, P. Journal of Computational Chemistry 2001, 22, 1048-1057.

(31) Cozzolino, A. F.; Vargas-Baca, I. Crystal Growth and Design 2011, 11, 668-677.

(32) Frisch, M. J. et al. Gaussian 09, Revision B.01, Wallingford CT, 2009.

(33) Chai, J. D.; Head-Gordon, M. Physical Chemistry Chemical Physics 2008, 10, 6615-6620.

(34) Weigend, F.; Ahlrichs, R. Physical Chemistry Chemical Physics 2005, 7, 3297-3305.

(35) Weigend, F. Physical Chemistry Chemical Physics 2006, 8, 1057-1065.

(36) Cornell, W. D.; Cieplak, P.; Bayly, C. I.; Kollman, P. A. Journal of the American Chemical Society 1993, 115, 9620-9631.

(37) R Core Team R: A Language and Environment for Statistical Computing, Vienna, Austria, 2019.

(38) Bleiholder, C.; Werz, D. B.; Köppel, H.; Gleiter, R. Journal of the American Chemical Society 2006, 128, 2666-2674.

(39) Bleiholder, C.; Gleiter, R.; Werz, D. B.; Köppel, H. Inorganic Chemistry 2007, 46, 2249-2260. 
(40) Cockroft, S. L.; Hunter, C. A. Chemical Society Reviews 2007, 36, 172-188.

(41) Bukowski, R. et al. SAPT2020: An Ab Initio Program for Many-Body Symmetry-Adapted Perturbation Theory Calculations of Intermolecular Interaction Energies, University of Delaware and University of Warsaw, 2020.

(42) Jeziorski, B.; Moszyński, R.; Szalewicz, K. Chem. Rev. 1994, 94, 1887-1930.

(43) Parrish, R. M. et al. Journal of Chemical Theory and Computation 2017, 13, 3185-3197.

(44) Capper, M. J.; Wright, G. S.; Barbieri, L.; Luchinat, E.; Mercatelli, E.; McAlary, L.; Yerbury, J. J.; O’Neill, P. M.; Antonyuk, S. V.; Banci, L.; Hasnain, S. S. Nature Communications 2018, 9, 1-9.

(45) Strange, R. W.; Antonyuk, S. V.; Hough, M. A.; Doucette, P. A.; Valentine, J. S.; Hasnain, S. S. Journal of Molecular Biology 2006, 356, 1152-1162. 